\begin{tabular}{|c|c|}
\hline Citation & $\begin{array}{l}\text { Anthony Coyette, Baris Esen, Ronny Vanhooren, Wim Dobbelaere, Georges } \\
\text { Gielen (2016) } \\
\text { Automatic test signal generation for mixed-signal integrated circuits by } \\
\text { circuit partitioning and interval analysis. } \\
\text { IEEE, International Test Conference (ITC) }\end{array}$ \\
\hline Archived version & $\begin{array}{l}\text { Author manuscript: the content is identical to the content of the published } \\
\text { paper, but without the final typesetting by the publisher. }\end{array}$ \\
\hline Published version & http://dx.doi.org/10.1109/TEST.2016.7805867 \\
\hline Journal homepage & http://www.itctestweek.org \\
\hline Author contact & $\begin{array}{l}\text { anthony.coyette@esat.kuleuven.be } \\
\text { + } 32 \text { (0)16 } 321159\end{array}$ \\
\hline IR & https://lirias.kuleuven.be/handle/123456789/576274 \\
\hline
\end{tabular}




\title{
Automatic Test Signal Generation for Mixed-Signal Integrated Circuits using Circuit Partitioning and Interval Analysis
}

\author{
Anthony Coyette ${ }^{1}$, Baris Esen ${ }^{1}$, Wim Dobbelaere ${ }^{2}$, Ronny Vanhooren ${ }^{2}$ and Georges Gielen ${ }^{1}$ \\ 1 Department of Electrical Engineering, KU Leuven, Belgium, \\ \{anthony.coyette, baris.esen, georges.gielen\}@esat.kuleuven.be \\ 2 ON Semiconductor Belgium, \\ \{wim.dobbelaere, ronny.vanhooren\}@onsemi.com
}

\begin{abstract}
A method is presented to address the automatic generation of test signals for analog and mixed-signal integrated circuits. No restriction on the number of inputs or the nonlinearity of the circuit are made. The circuit under consideration is first decomposed into a set of sub-circuits, called blocks, in order to break down the complexity of the problem. The effect of a targeted fault is then automatically analyzed at the transistor level in a defect-oriented context. From this analysis, the fault sensitization conditions are extracted and then backtraced towards the primary inputs or outputs of the circuit using an algorithm based on the interval analysis theory. The underlying algorithms supporting the automation of the whole procedure are illustrated for basic circuits. Finally, in order to demonstrate the method, an industrial circuit is used as case study. It is shown that test signals can be generated in order to achieve a fault coverage of $98 \%$.
\end{abstract}

\section{Introduction}

The quality requirements for integrated circuits manufactured by the silicon industry have been increasing during the last decades. For instance, the automotive industry is nowadays combining around 400 integrated circuits (ICs) per vehicle and this number is expected to keep growing the next coming years. As a result, the expected defect level per IC has broken through the part-per-million barrier and the actual automotive industry expectation is now of the order of 100 parts-per-billion or lower. Furthermore, with an ever-shortening time-to-market, pressure is put on the time allocated to the test design. Therefore, an automation as well as an improvement of the test development are desired.

In the case of digital circuits, both the test pattern generation guaranteeing a defined quality level and the automation of this generation have been solved. Algorithms such as the D-algorithm or FAN have paved the way to this desired automation [1], [2]. The required quality can be achieved thanks to the use of a test approach involving scan insertion and a combination of stuck-at, bridging, Iddq and transition ATPG patterns.

Nowadays, the majority of the field failures in mixedsignal automotive ICs can be traced back to defects in the analog part [3]. This comes due to the fact that the analog circuitry is typically tested in an empirical way based on the functional and performance specifications. And the question of the automated test signal generation for analog circuits remains alive. It has been addressed in literature but the desired requirements still cannot be met in daily practice. Several methods have been proposed during the last decades but these suffer from drawbacks such as the poor scalability with the number of inputs these can cope with or the limited nonlinearity allowed for the circuit under consideration. In general, these methods cannot cope with the complexity of an industrial circuit presenting high nonlinearity and several inputs for configurations such as pull-down transistors.

The limitation of the number of allowed inputs arises from the common practice to consider a nonlinear circuit as a blackbox. In studies such as [4] and [5], the inputoutput relationship is used and the signal generation is approached as an optimization of the input stimuli in order to maximize the difference between the output of a good circuit and a faulty one. This leads to searching for input stimuli in the input space which increases exponentially with the number of inputs. Therefore, these techniques suffer from a limitation on the number of inputs allowed for the circuit under consideration.

This limitation can be overcome by exploiting information about the circuit during the signal generation. This approach has been adopted in [6] where the Signal Flow Graph method is introduced and the circuit is represented by a graph of interconnected impedances. Later, the concept of Testability Analysis has been introduced and has pushed further the analysis by deriving new values from the impedances [7] [8]. But as already stated, the nonlinearity these techniques can handle is limited.

In this paper, a method is introduced to automatically generate a test signal targeting a given fault for a given analog or mixed-signal circuit. By breaking the circuit into sub-circuits, the exponential complexity as a function of the number of inputs has been solved. The sub-circuits are modeled such that no limitation is imposed on their nonlinearity. And, based on these model, a calculus method based on interval analysis is introduced in order to generate 


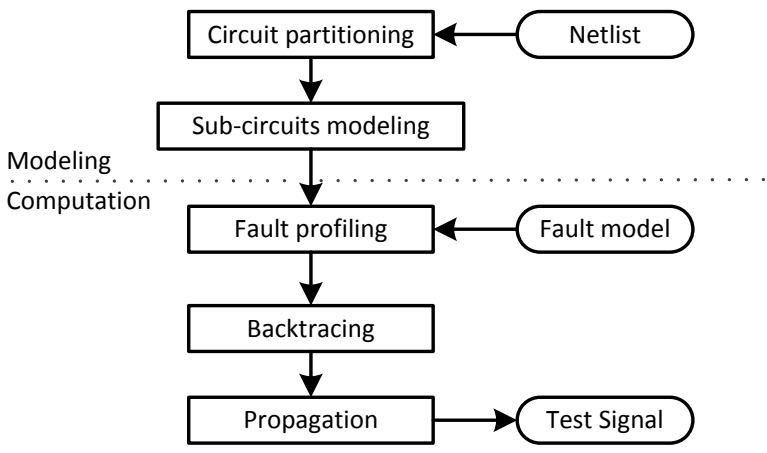

Figure 1: Flow diagram of the proposed automatic test generation method.

test signals. The work paradigm of the method is introduced in Section 2. Then, the overall structure of the method is outlined in Section 3 and put in perspective with the testing of digital ICs. In Section 4 and Section 5, the two main phases of the proposed method are explained in detail. The underlying algorithms are presented and illustrated for small example circuits. Afterwards, considerations about the method and possible further developments are discussed in Section 6. Simulations results for an industrial circuit are presented in Section 7. Finally, conclusions are drawn in Section 8 .

\section{Work paradigm}

The method proposed in this paper follows the defectoriented approach. Therefore, a small overview is first given. On that basis, the concept of fault-centered approach is introduced as an extension.

\subsection{Defect-Oriented Method}

The literature on the testing of analog integrated circuits makes numerous references to the defect-oriented approach [9]-[11]. In contrast to the specification-based approach, where the targeted performances of a circuit are tested, the defect-oriented methodology aims at testing for the presence of a defect. To that end, the possible defects which can occur in that circuit are represented by faults according to fault models and their effects on the circuit can be simulated at the transistor level.

Defects occurring in analog integrated circuits are generally divided into two categories: parametric defects and catastrophic defects. In the scope of this work, parametric defects are not discussed and the focus is set on catastrophic defects. These defects emerge from a problem during the silicon manufacturing process such as a dust particle, an under-etching, etc., causing a change in the topology of the manufactured circuit. These defects can be modeled by open and short circuits such as it is done in the 5-fault model adopted in this work [12].

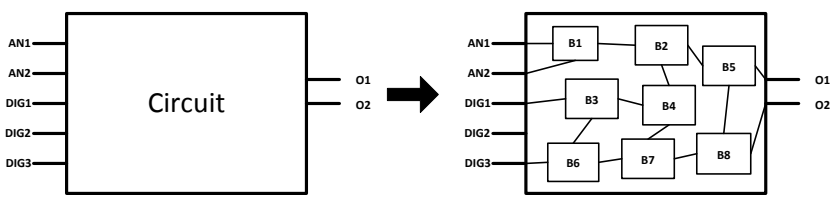

Figure 2: A circuit is seen as a set of interconnected blocks.

\subsection{Fault-Centered Approach}

In this work, the concept of fault-centered approach is introduced as an extension of the defect-oriented approach. This terminology is used to emphasize the specificity of the method proposed in this paper in comparison to a general defect-oriented method. Indeed, many studies that have adopted the defect-oriented method proceeded to the maximization of the fault coverage for a given circuit. However, only a few techniques have focused on the generation of test signals targeting a specific fault.

The distinction in the process of test generation appears as being important in the context of the recent developments in the field of analog testing. With the arrival of software enabling the automated computation of fault coverages [11], [13], it becomes possible to integrate a quality assessment step in the design flow of analog integrated circuits. The test designer knows to what extent an IC is actually tested and the list of faults which are not yet detected by the test program at hand is made available. Therefore, if test generation methods can be developed in order to target specifically given faults, the whole test design workflow could be further advanced.

\section{Proposed Test Generation Method}

The method presented in this paper follows the faultcentered approach introduced in the previous section. It aims at generating a test stimulus for a given circuit and a given fault in an automated way. The overall workflow can be decomposed into the series of successive steps illustrated in the flow diagram of Figure 1. In this section, the global architecture of the method is addressed and a comparison is made with the testing of digital circuits. The details of the implementation will be given in the following sections.

Starting from the circuit netlist, the method involves two main phases: a modeling phase and a computation phase. The general idea can be put into perspective with the way digital ATPG methods are developed today in industry.

During the modeling phase, the core idea is to model the considered circuit in order to enable the second phase i.e. the computation phase. This phase needs to be done only once for the considered circuit. Based on the circuit netlist, the procedure starts with the circuit partitioning. During that step, the circuit is divided into sub-circuits as illustrated in Figure 2. In the following, these sub-circuits are referred to as blocks. Each of these blocks presents maximum three terminals in order to break down the complexity of the problem. Indeed, when a circuit is considered as a blackbox, 
its input space grows exponentially with the number of inputs. By limiting the number of terminals for each block, the input space of each block is limited and it can be easily modeled. In other words, the problem is transformed into a set of blocks interacting with each other according to Kirchhoff's laws.

In the case of digital circuits, this phase does not happen explicitly since the decomposition is naturally present due to the way digital circuits are built from digital gates. Also, the models of the used digital gates are already known and do not require extra modeling. It is worth noting that most of the analog circuits are also built with a restricted number of building blocks such as current mirrors or differential pairs. Therefore, a part of the blocks modeling could be avoided by building a library of blocks for which a model has already been built. This point will be discussed later.

During the computation phase, the core idea is similar to the digital workflow. Starting from a simple fault model such as the stuck-at fault model, the impact of a fault on a digital circuit is studied by means of the 5 -valued algebra [2]. This algebra introduces the values $\mathrm{D}$ and $\overline{\mathrm{D}}$ which represent the difference between the good and the faulty circuit. Then, this value D is backtraced towards the primary inputs and propagated towards the primary outputs. Similarly, it is here proposed to inject a catastrophic fault into the circuit according to a fault model such as the 5-fault model [12]. The impact of the fault on the circuit is evaluated at the local level and the circuit conditions for which these effects can be observed are extracted. Then, these internal conditions have to be backtraced and propagated to the primary inputs/outputs. However, the backtracing and propagation mechanisms are not as straightforward in analog circuits as in the digital case where circuits exhibit a clear directionality. In order to tackle this problem, the algorithm presented in the following are based on interval analysis. In the developments, no specific hypothesis is made about the circuit under test. Therefore, the method can be applied to any type of analog integrated circuit.

\section{Phase I: Circuit Modeling}

The first part of the proposed method consists in the modeling of the considered circuit in order to enable the computations in the second phase. The circuit is divided into blocks and each block is modeled such that it can be handled easily and efficiently during the computations. These two operations are called the circuit partitioning and the blocks profiling and are detailed in the following.

\subsection{Circuit partitioning}

The partitioning of the circuit is introduced in order to break down the complexity of the problem. The circuit under consideration is decomposed into a number of interconnected blocks; each presenting maximum three terminals, such as illustrated in Figure 2.

The goal is therefore to divide the initial circuit into a minimum number of blocks. A minimum number of

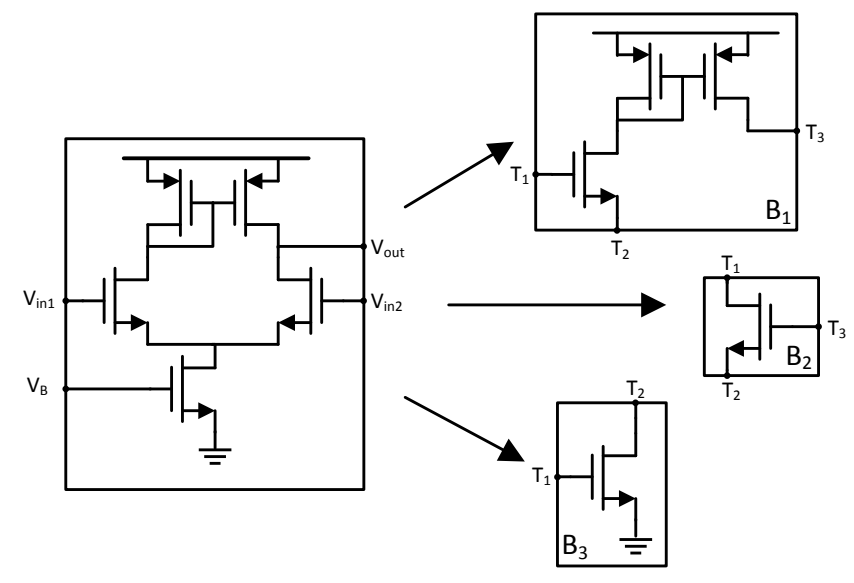

Figure 3: Circuit partitioning for a differential amplifier circuit.

blocks is desirable for both the modeling phase and the computation phase. Indeed, a reduced number of blocks means that less time needs to be spent on the simulations in order to build the models, and a reduced number of blocks means in general a smaller number of interconnection nodes for the computations. Considering that the method relies on the resolution of the Kirchhoff's current law at these interconnection nodes, a smaller number of nodes means a smaller system of equations.

This combinatorial optimization is carried out by a genetic algorithm [14]. The result for a differential amplifier is illustrated in Figure 3. It is worth noting that the power supply voltage and the ground are not considered as terminals since they are not variables of the system. In the case where the power supply voltage can be controlled inside the integrated circuit [15] and is therefore variable, it can be considered as a terminal and be part of the test signal generation. However, this is not done in the scope of this paper.

\subsection{Blocks Profiling}

The blocks resulting from the circuit partitioning have to be modeled in order to be used during the second phase of the method i.e. the computation phase where the test signals are generated. As will be explained in the next section, the test generation makes use of the interval analysis theory. This point is relevant in the selection of the models to use. The I-V relationships of the blocks need to be available such that the current through the terminals of a block $B_{i}$ can be calculated as a function of the voltages at these terminals. And inversely, the model should be able to give the voltages at the terminals of the block for a given current flowing through a terminal. Furthermore, these calculations should be compatible with the interval arithmetic that will be introduced later.

In the scope of this work, piecewise linear approximations have been used to model the $\mathrm{I}-\mathrm{V}$ relationships of a block. Other models, such as Differential Algebraic Equations (DAE), are also compatible with the proposed 


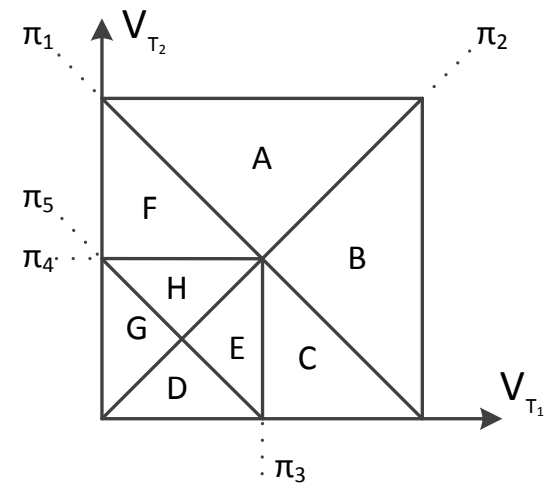

(a) Domain of the approximation:

simplicial mesh obtained by binary refinement.

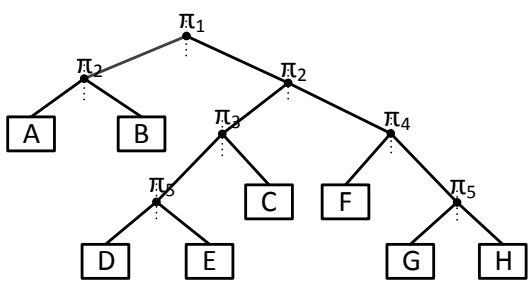

(b) Data-structure:

binary space partitioning tree.

Figure 4: Two visualizations of the binary refinement mechanism.

method. These could be used as a refinement to get a faster implementation. The piecewise linear approximations were chosen here for their relative simplicity.

As stated above, a model has to be built for each terminal of each block so that the current can be calculated as a function of the terminal voltages. The terminology $I_{B_{i}, T_{j}}$ designates the piecewise linear function approximating the current flowing through the terminal $T_{i}$ of the block $B_{j}$. For instance, the block $B_{3}$ from Figure 3 presents the two terminal $T_{1}, T_{2}$, and therefore two models have to be built: $I_{B_{3}, T_{1}}\left(v_{1}, v_{2}\right)$ and $I_{B_{3}, T_{2}}\left(v_{1}, v_{2}\right)$ with $v_{1}, v_{2} \in V_{0}$ where $V_{0}$ is the interval $[0, \mathrm{Vdd}]$ representing the voltage range that the circuit nodes can assume; Vdd being the power supply voltage.

The construction of these models is based on transistorlevel simulation data. In order to achieve an accuracy predefined by the user, a binary refinement scheme is implemented [16] and the simulation data are produced online during the refinement process. In this paragraph, the developments are explained for the example of a 2-terminal block i.e. a piecewise linear approximation of two variables. Starting from the approximation domain being the interval $V_{0} \times V_{0}$, the initial decomposition consists of two triangles which are simplices in 2 dimensions. Then, each simplex is tested to verify if it approximates the block current sufficiently accurately according to a chosen threshold. If the simplex does not accurately approximate the targeted function, it is refined through a bisection i.e. the simplex is decomposed into two new simplices. The result of this

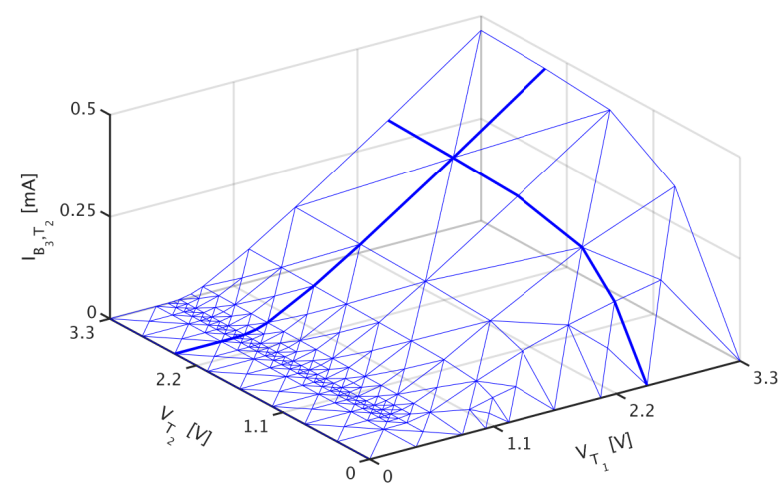

Figure 5: Piecewise linear model of the 2-terminal block $B_{3}$.

modeling process is illustrated in Figure 4. In Figure 4a, the initial 2-dimensional interval $V_{0} \times V_{0}$ is shown with such a decomposition. The 2-dimensional interval is initially split into two triangles by the diagonal $\pi_{1}$ of the square. The same happens to the upper simplex which is split into two simplices by the diagonal $\pi_{2}$. Figure $4 \mathrm{~b}$ illustrates the binary space partitioning tree which is built by this procedure [17]. In the 3-dimensional case, the same can be done by splitting the initial interval, a cube, into six tetrahedra. The decomposition into triangles in the 2-dimensional case and tetrahedra in the 3-dimensional case is called a simplicial meshing of the space. This process of binary refinement presents the advantage that it locally approximates the targeted function up to the desired precision. Furthermore, by carefully proceeding to the iterative bisection of the simplices, the simplicial meshing can be done such that the piecewise linear approximations are continuous functions [16].

A model built for the block $B_{3}$ of the differential amplifier example is illustrated in Figure 5. The block consists of a single MOSFET transistor from which the source is grounded. Therefore, the built model $I_{B_{3}, T_{2}}$ has a familiar shape since it exhibits the current flowing through the drain of a nMOS transistor as a function of $V_{G S}$ (or $V_{T_{1}}$ ) and $V_{D S}$ (or $V_{T_{2}}$ ). Bold lines in Figure 5 highlights the recognizable curves.

\section{Phase II: Automatic Test Signal Generation}

Based on the piecewise linear approximations built for each block during the modeling phase, the actual generation of a test signal able to detect a given fault consists of three steps: the fault sensitization, the backtracing step and the propagation step. In the scope of this paper, most focus is set on the backtracing mechanism of DC signals.

\subsection{Fault sensitization}

The fault sensitization step consists in finding the effects of the given fault $F$ when it is present in the circuit. As 
introduced above, the effects of this fault $\mathrm{F}$ are assessed locally, meaning that the fault is injected into the block containing the defective transistor. Without loss of generality, the following developments are expressed for a 2-terminal block $B_{i}$. The injection of the fault $\mathrm{F}$ into $B_{i}$ delivers a new 2-terminal block $B_{i}^{\prime}$. The terminal currents are remodeled with the added fault and the two new piecewise linear models $I_{B_{i}^{\prime}, T_{1}}$ and $I_{B_{i}^{\prime}, T_{2}}$ are created.

The comparison of the faulty models $I_{B^{\prime}, T_{1}}$ and $I_{B^{\prime}, T_{2}}$ to the fault-free ones, $I_{B, T_{1}}$ and $I_{B, T_{2}}$, leads to defining a region $D$ where a difference between the behavior of the blocks is detected. In mathematical words, the fault sensitization region $\mathrm{D}$ is defined as:

$$
D=\left\{\mathbf{v} \in V_{0} \times V_{0} \mid \bigvee_{j=1,2} d\left(I_{B_{i}, T_{j}}(\mathbf{v}), I_{B_{i}^{\prime}, T_{j}}(\mathbf{v})\right)>\alpha\right\}
$$

where $V_{0}$ is the voltage interval $[0, \mathrm{Vdd}], \mathrm{d}$ is a difference metric function and $\alpha$ is a chosen parameter determining the minimum difference required to be selected. In this paper, the simulations are carried out with $d(a, b):=\|a-b\|$ being the current difference and $\alpha=150 \mu A$. Furthermore, a simplification is made for the construction of the sensitization region $D$ based on the piecewise linear approximations. Instead of exactly constructing the region by comparing the piecewise linear approximations, only the center of the linear pieces are compared. As a result, a set of points $\left\{\mathbf{v}_{\mathbf{1}}, \ldots, \mathbf{v}_{\mathbf{n}}\right\}$ with $\mathbf{v}_{\mathbf{i}} \in V_{0}^{2}$ is found which approximates the sensitization region.

In a second step, the region defined by the set of points is transformed into a set of intervals such that the information can be exploited during the following steps, i.e. it complies to the interval analysis. In the scope of this work, the transformation of the fault sensitization region into intervals happens in two sub-steps. First, the set of points is divided into the separated clusters of points. Then, for each cluster, the minimum axis-aligned bounding box is found [18].

Figure 6 gives an illustrative example of the fault sensitization for the faulty transmission gate pictured in Figure 6a. In order to keep the problem in two dimensions for visualization purposes, the transmission gate has been closed by connecting the gates of the nMOS (resp. pMOS) transistors to ground (resp. Vdd). The added resistor models a drainsource short fault on the nMOS transistor and is attributed a value of $10 \mathrm{k} \Omega$. After the fault sensitization analysis, it is found that a different behavior can be observed for this transmission gate when one of the terminals has a voltage close to Vdd and the other one a voltage close to ground. This can be seen in Figure $6 \mathrm{~b}$ where two clusters are present and each of them is enclosed by a minimum axis-aligned bounding box.

\subsection{Backtracing step}

The goal of the backtracing step is to find the signals that have to be applied on the primary inputs/outputs of the considered circuit in order to drive it in the region $D$ calculated during the fault sensitization. By doing so, a

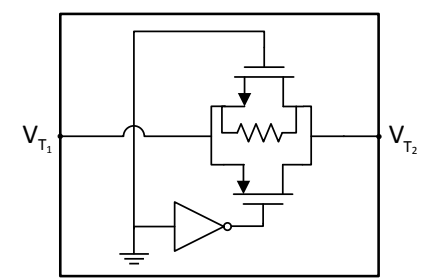

(a) Schematic circuit of the closed transmission gate with a drain-source short fault.

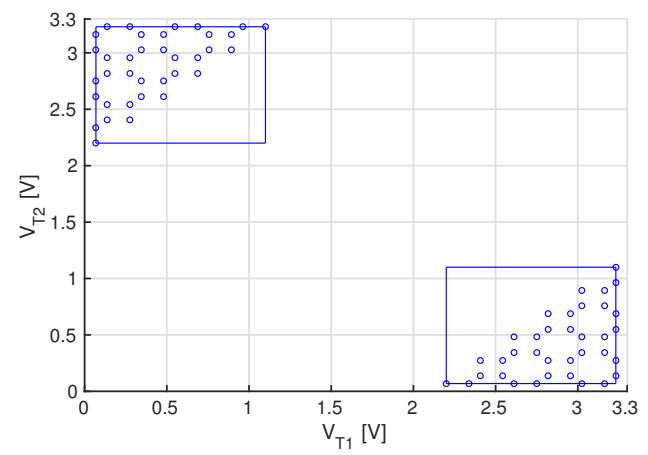

(b) The region $D$ consists of 2 clusters, each defined by a minimum axis-aligned bounding box.

Figure 6: Illustration of the fault sensitization step for the example of a defective transmission gate.

different behavior in case of faulty circuit can be activated locally.

In order to solve the backtracing problem, the proposed method uses the interval analysis theory [19]. In the field of electronics, this theory has already been successfully applied in order to find all DC solutions of nonlinear analog circuits [20]. In the following, it is explained how test signals can be generated by developing the Kirchhoff's current law at the nodes connecting the blocks of the circuit and by solving the system of equations with interval analysis. A small introduction of the used mathematical tools is first given. Then, the application of these concepts to the backtracing problem are presented and illustrated by simple examples.

5.2.1. Interval Analysis. The interval analysis theory is a framework that considers problems in terms of intervals instead of real numbers. The notation $[X]=$ $[a, b]$ designates the interval containing the set of reals $\{x \in \mathbb{R} \mid a<x<b$ with $a, b \in \mathbb{R}\}$ where $\mathbb{R}$ denotes the set of real numbers. A specific arithmetic is dedicated to these intervals with the four basic operations i.e. addition, subtraction, multiplication and division. For instance, the addition of two real intervals $\left[X_{0}\right]=\left[a_{0}, b_{0}\right]$ and $\left[X_{1}\right]=\left[a_{1}, b_{1}\right]$ is defined as:

$$
\left[X_{0}\right]+\left[X_{1}\right]=\left[a_{0}, b_{0}\right]+\left[a_{1}, b_{1}\right]=\left[a_{0}+a_{1}, b_{0}+b_{1}\right]
$$

where $a_{0}, a_{1}, b_{0}, b_{1} \in \mathbb{R}$.

In addition to this interval arithmetic, the interval analysis theory also offers more advanced tools handling intervals. Two of them are used in the backtracing mechanism and are 
introduced in the following: contractors and the Branch-andBound algorithm.

A contractor $\mathcal{C}$ is an operator projecting an interval $[\mathrm{x}]$ onto another interval $\mathcal{C}([\mathrm{x}])$ such that $\mathcal{C}([\mathrm{x}]) \subset[\mathrm{x}]$. Several types of contractor exist and are documented in literature. The reader is referred to the literature for details about these contractors [19]. In the scope of this paper, only two different contractors are introduced and used by the method. The first kind of contractors used is based on the concept of constraint satisfaction problem. The basic idea is to transform an equation (or constraint) into a contractor. For instance, the equation $x_{1}+x_{2}-x_{3}=0$ can be transformed into three contractors by isolating each of the involved variables. One of these contractors is:

$$
\mathcal{C}_{C}:\left[x_{1}\right] \times\left[x_{2}\right] \rightarrow\left[x_{3}\right] \cap\left(x_{1}+x_{2}\right)
$$

which can also be written as:

$$
\phi\left(\text { in: } x_{1}, x_{2} ; \text { out: } x_{3}\right)\left\{x_{3}:=x_{1}+x_{2}\right\}
$$

The second kind of contractor used is based on the Newton-Raphson method for solving nonlinear equations and the rewriting of its main formula into the interval analysis framework. In the case of a nonlinear function $f$ of one variable, the Newton-like contractor for the equation $f(x)=0$ can be written as:

$$
\mathcal{C}_{N}:[x] \rightarrow[x] \cap\left(x-\frac{f([x])}{f^{\prime}([x])}\right) .
$$

This type of contractor can also be extended to multivariate functions.

In the following, the action of these contractors is illustrated for the example of a voltage divider. Developments are limited to the constraint satisfaction contractors for concision purposes. The voltage divider circuit:

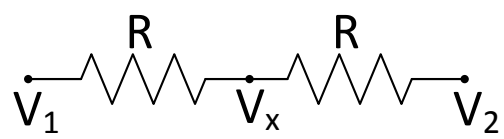

is considered where $\mathrm{V}_{1}, \mathrm{~V}_{2}, \mathrm{~V}_{x}$ are the node voltages and $\mathrm{R}$ is the value attributed to the two resistors. The equation describing this circuit can be written as:

$$
V_{\mathrm{x}}=V_{1}+\frac{R}{R+R}\left(V_{2}-V_{1}\right)=\frac{V_{2}+V_{1}}{2} .
$$

Based on this equation and the contractors introduced previously, a partial analysis of the circuit can be done. For instance, assuming that the voltage $\mathrm{V}_{\mathrm{x}}$ is requested to be $2 \mathrm{~V}$, the set of values for $\mathrm{V}_{1}$ and $\mathrm{V}_{2}$ fulfilling this condition can be searched. The solution to this question is not unique and can be treated as follows. First, boundaries have to be set for the considered problem. In this example, it is assumed that the node voltages $V_{1}$ and $V_{2}$ should be in the range from $0 \mathrm{~V}$ to $3 \mathrm{~V}$. Hence, the initial search domain $V_{0}=$ $\left[V_{1}\right] \times\left[V_{2}\right]=[0,3] \times[0,3]$ is fixed. Then, by isolating $V_{2}$, the contractor:

$$
\phi\left(\text { in: } V_{1} ; \text { out: } V_{2}\right)\left\{V_{2}:=4-V_{1}\right\}
$$

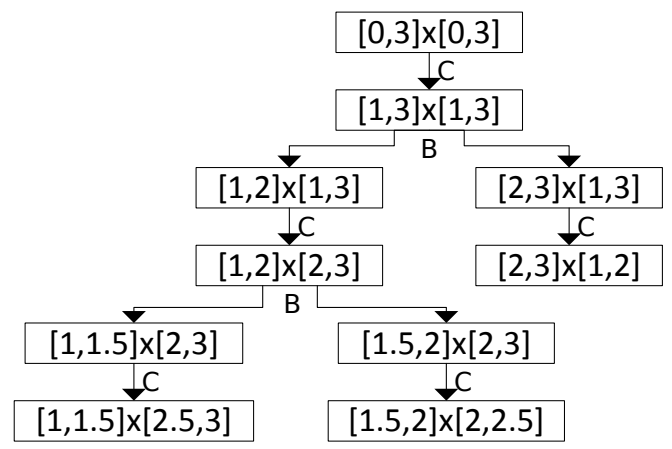

(a) Successive contractions (C) and bisections (B).

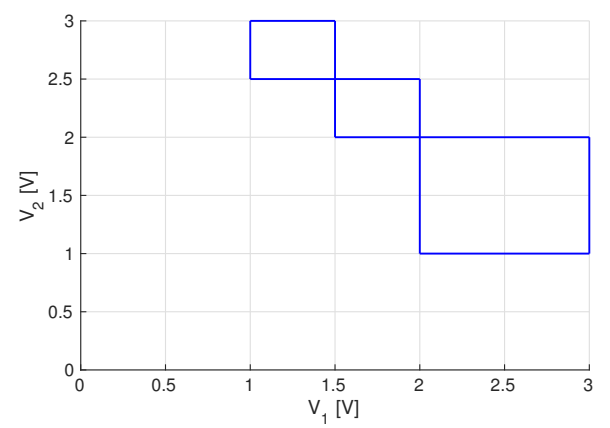

(b) $2 \mathrm{D}$ representation of the leafs in the search tree.

Figure 7: Visualization of the Branch-and-Bound algorithm.

can be constructed and the initial interval can be contracted into $[1,3] \times[0,3]$, reducing the range of possible values for $V_{2}$. By symmetry, a contractor can be found for $V_{1}$ and the same contraction can be realized.

However, once the interval becomes $[1,3] \times[1,3]$, it cannot be contracted further. To cope with that limitation, the proposed method makes use of another tool from the interval analysis theory: the Branch-and-Bound (BB) algorithm. When an interval cannot be further contracted, this one is bisected i.e. divided into two intervals. By combining interval contractors and bisections, the BB algorithm allows to solve systems of nonlinear equations [21]. The main idea of this BB algorithm consists in consecutively contracting an interval and then bisecting it into two sub-intervals. By repetitively alternating these contractions and bisections, a tree searching the initial interval is created. Such a tree can be seen in Figure $7 \mathrm{a}$ where the BB algorithm has been terminated after a few iterations for the voltage divider example. In general, the recursive process continues until the size of the interval reaches a precision $\lambda$ determined by the user. This process is summarized by Algorithm 1 which shows the pseudo-code of the recursive formulation of the approach. Figure 8 illustrates the final solution obtained at the end of the BB algorithm.

5.2.2. Backtracing in nonlinear circuits. Based on the interval analysis tools that were presented in the previous 

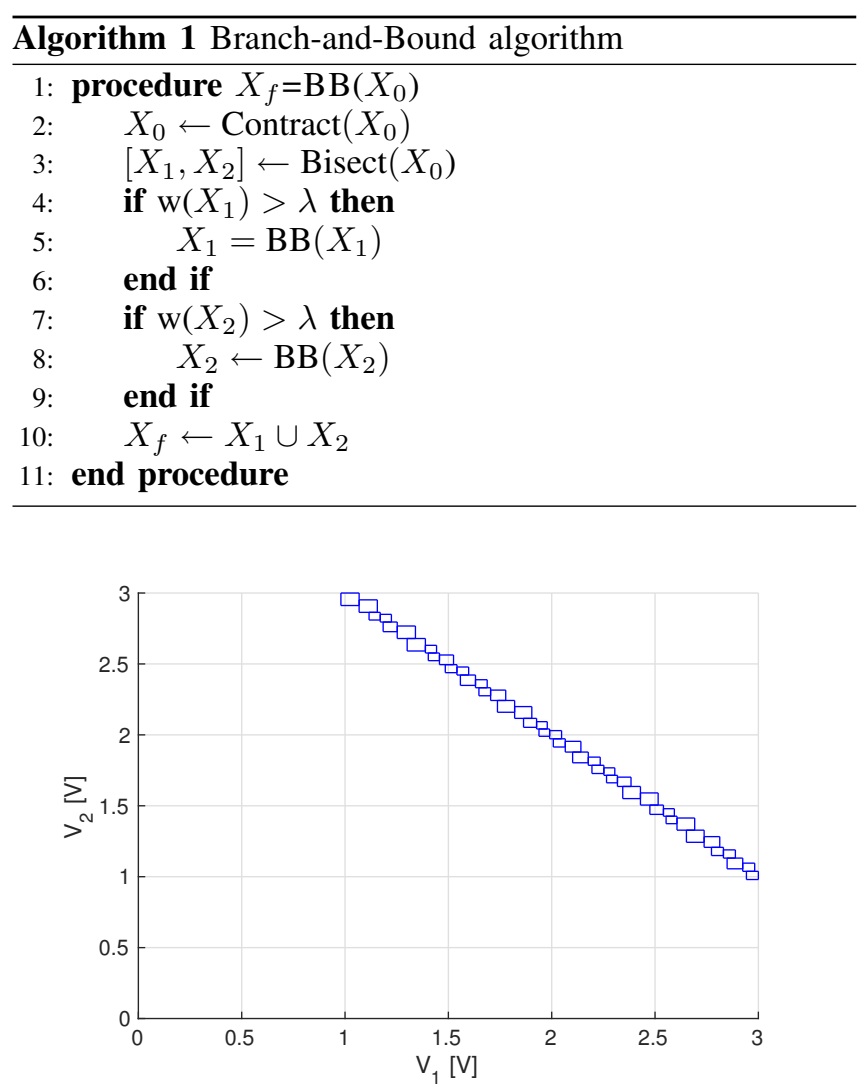

Figure 8: Interval solution to the backtracing problem for the voltage divider $\left\{\left(V_{1}, V_{2}\right) \in[0,3]^{2} \mid V_{x}=2 V\right\}$.

section and the built piecewise linear approximations, the test signal generation problem can be addressed. In the following, details are given about the way these tools are used in the proposed method. The developments are expressed for the example of the differential amplifier but the same can be done automatically for any circuit.

Kirchhoff's current law is expressed for each node which is nor an input nor an output. Then, a set of contractors is generated based on these equations. For the differential amplifier, Kirchhoff's current law is applied to the common mode node and the current mirror node and gives the two equations:

$$
\begin{array}{r}
I_{B_{1}, T_{2}}+I_{B_{2}, T_{2}}+I_{B_{3}, T_{2}}=0 \\
I_{B_{1}, T_{3}}+I_{B_{2}, T_{1}}=0
\end{array}
$$

These two equations deliver 5 contractors:

$$
\begin{aligned}
& \mathcal{C}_{1}\left(\text { in: } I_{B_{1}, T_{2}}, I_{B_{2}, T_{2}} ; \text { out: } I_{B_{3}, T_{2}}\right)\left\{I_{B_{3}, T_{2}}:=-I_{B_{1}, T_{2}}-I_{B_{2}, T_{2}}\right\} \\
& \mathcal{C}_{2}\left(\text { in: } I_{B_{1}, T_{2}}, I_{B_{3}, T_{2}} ; \text { out: } I_{B_{2}, T_{2}}\right)\left\{I_{B_{2}, T_{2}}:=-I_{B_{1}, T_{2}}-I_{B_{3}, T_{2}}\right\} \\
& \mathcal{C}_{3}\left(\text { in: } I_{B_{2}, T_{2}}, I_{B_{3}, T_{2}} ; \text { out: } I_{B_{1}, T_{2}}\right)\left\{I_{B_{1}, T_{2}}:=-I_{B_{2}, T_{2}}-I_{B_{3}, T_{2}}\right\} \\
& \mathcal{C}_{4}\left(\text { in }: I_{B_{1}, T_{3}} ; \text { out: } I_{B_{2}, T_{1}}\right)\left\{I_{B_{1}, T_{3}}:=-I_{B_{2}, T_{1}}\right\} \\
& \mathcal{C}_{5}\left(\text { in }: I_{B_{2}, T_{1}} ; \text { out: } I_{B_{1}, T_{3}}\right)\left\{I_{B_{2}, T_{1}}:=-I_{B_{1}, T_{3}}\right\}
\end{aligned}
$$

The initial interval is constructed by allocating an interval $[0, \mathrm{Vdd}]$ to all circuit nodes, with Vdd being the power

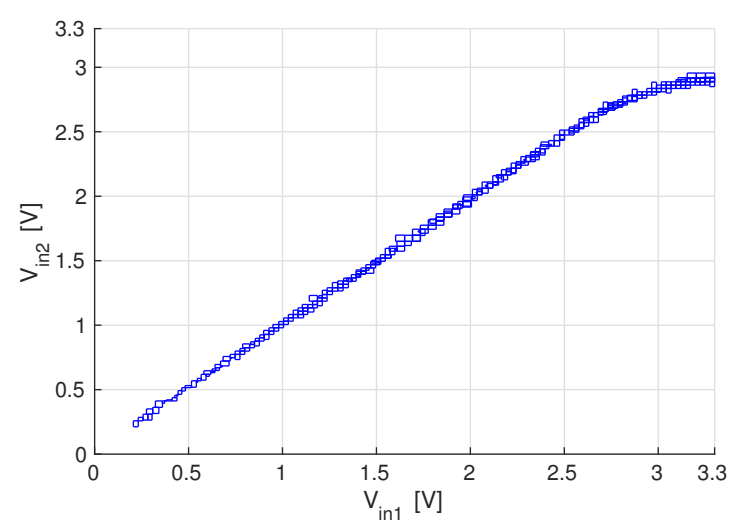

Figure 9: Interval solution to the problem for the differential amplifier $\left\{\left(V_{\text {in } 1}, V_{\text {in } 2}\right) \in[0, \mathrm{Vdd}]^{2} \mid V_{\text {out }}=2 \mathrm{~V}\right\}$.

supply voltage of the circuit. Then, the information resulting from the fault sensitization step is used to modify the initial interval before starting the backtracing process. During the fault sensitization, the region $D$ ensuring the local sensitization of the given fault $\mathrm{F}$ is extracted and transformed into an interval (or several intervals) for the block terminals. This interval determines the initial interval for the circuit nodes corresponding to the terminals of the block. Furthermore, the solution can be facilitated by constraining a node to a value $V_{c}$. This is done by attributing an interval $\left[V_{c}-\epsilon, V_{c}+\epsilon\right]$ to this circuit node where $\epsilon$ is chosen arbitrarily small.

The actual backtracing process can then start. The Branch-and-Bound algorithm is applied to the initial interval modified by the information found during the fault sensitization step. The contractions are operated by calculating the contractors expressions. These calculations are carried out based on the piecewise linear approximations built during the modeling phase. The previously explained succession of contractions and bisections continues until the precision $\lambda$ is reached. The solution found gives which signals should be imposed on the circuit inputs/outputs so that the given fault $F$ is locally activated. It is worth noting that the interval analysis finds all existing solutions to a problem and therefore offers a decisive answer to the question whether the circuit can be tested with DC stimuli without additional Design-for-Testability (DfT). If no solution can be found, it can be affirmed that the testing of this defect requires extra DfT or non-DC testing.

In order to demonstrate this backtracing process, a test example has been carried out for the differential amplifier shown in Figure 2. The backtracing has been carried out in order to request the set of voltages $\left(V_{i n 1}, V_{i n 2}\right)$ such that the output voltage is equal to $2 \mathrm{~V}$ with a power supply voltage of $3.3 \mathrm{~V}$ and a bias voltage of $1.2 \mathrm{~V}$. The result of this experiment is shown in Figure 9. The final result consists of a set of intervals paving the expected curve. 


$$
\sum_{\vdots}^{\frac{V d d}{5} R_{n}}
$$

(a) Linear circuit.

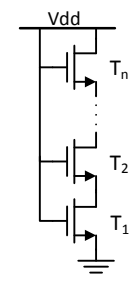

(b) Nonlinear circuit.
Figure 10: Schematic diagram of the benchmark circuits used to evaluate the time complexity of the method.

\subsection{Propagation step}

The propagation step consists in finding a test stimulus allowing to observe a difference between a faulty circuit and the good circuit. While the backtracing mechanism ensures to locally activate the fault, it does not ensure to make it observable at the circuit inputs/outputs. But as already introduced, the propagation in analog circuits is not as straightforward as in digital circuits. In the scope of this paper, it is proposed to monitor the results during the backtracing process and terminate it as soon as a test signal allowing to detect the fault is generated. As a future development, the current consumption of the circuit could be measured as a propagation mechanism and would facilitate the propagation step.

\section{Discussion}

The method proposed in the previous section rises a few points worth noting, as these discussed in the following.

\subsection{Scalability}

In order to evaluate the scalability of the method, experiments have been carried out with the benchmark circuits illustrated in Figure 10. These circuits are parametrizable in the sense that they consist of $n$ elements, where $n$ is a natural number bigger than 1 which can be chosen. By increasing this number $\mathrm{n}$ from 5 to 40 by step of 5 and measuring the runtime of the method, its time complexity was experimentally estimated.

The first experiment was carried out for the linear benchmark circuit illustrated in Figure 10a. This parametrizable circuit is a voltage divider presenting $\mathrm{n}$ resistors and hence $\mathrm{n}+1$ nodes. The backtracing algorithm was used to solve the circuit where the second node was set to a voltage of $0.5 \mathrm{~V}$. The time taken to calculate the whole solution for that problem was measured. The results of this experiment are shown in Figure 11a where the trend of the time complexity as a function of the number of nodes can be observed. It can be seen that this evolution happens exponentially. This comes from the fact that even for a linear circuit, the solution space grows exponentially with the number of nodes i.e. the number of intervals needed to pave the solution grows exponentially.

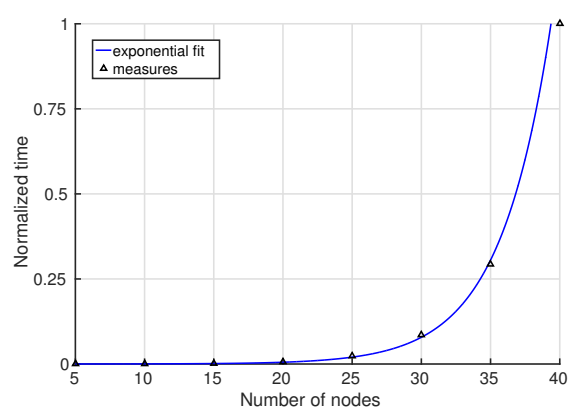

(a) Time for complete solution in linear circuits.

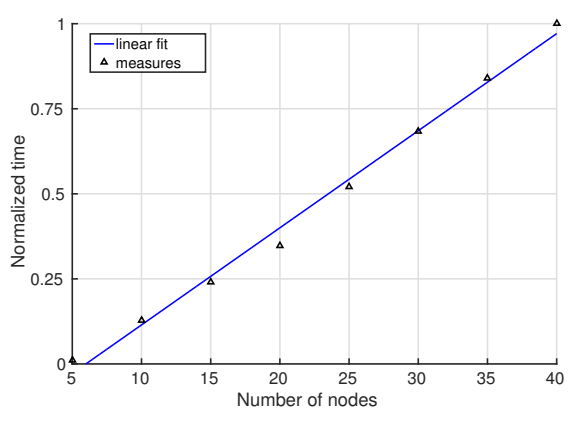

(b) Time before first solution in linear circuits

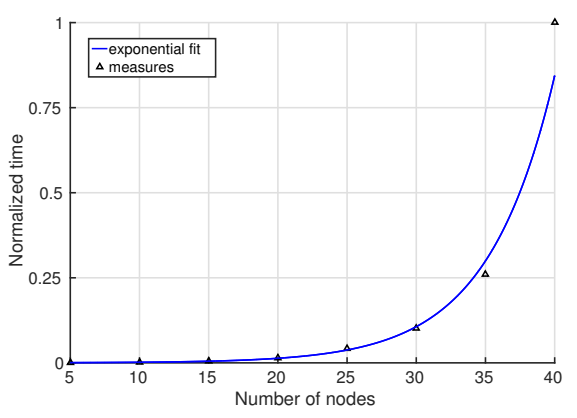

(c) Time before first solution in nonlinear circuits.

Figure 11: Experimental measures of the time complexity of the backtracing algorithm.

However, calculating the whole solution of such a problem is not the prime goal of this work. Indeed, only one working solution must be found in order to detect the fault. Therefore, in a second time, the time taken before finding the first solution was studied. This experiment was carried out for the linear and nonlinear benchmark circuits. The nonlinear benchmark circuit, illustrated in Figure 10b, consists of $\mathrm{n}$ transistors connected in series from which the gates are connected to the power supply voltage Vdd. From the results shown in Figure 11b-c, it can be seen that the time complexity evolves linearly for linear circuits and exponentially for nonlinear circuits.

The simulations show that this method is not meant to be applied to circuits which cannot be partitioned into a small amount of blocks. However, in contrast to this analysis, the case study presented in Section 7 demonstrates that results can be achieved for circuits presenting 50 transistors. Furthermore, the presented method is highly suitable for parallel 


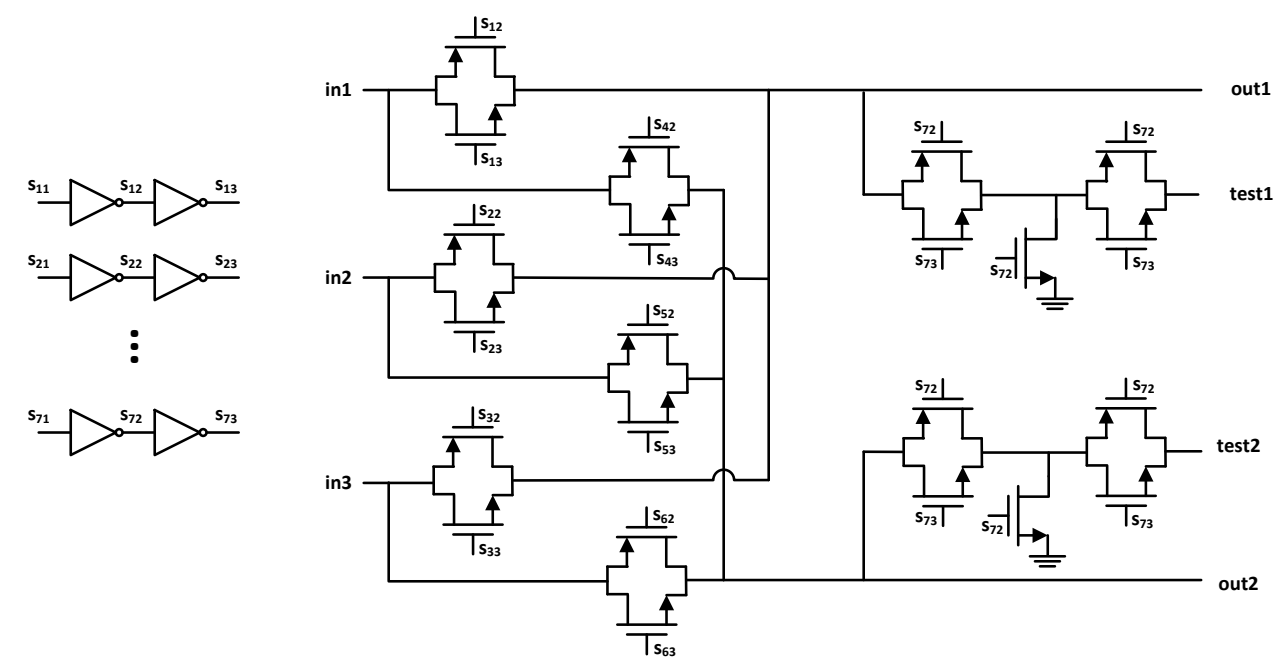

Figure 12: Schematic diagram of the analog multiplexer circuit used as case study.

implementations which would shorten the time needed to generate test signals. Therefore, the proposed technique is a good candidate for the automatic generation of test signal but might need further investigation in order to know its limitations and real potential.

\subsection{Blocks Profiling}

In order to proceed to the computation phase, a circuit first needs to be partitioned into blocks which then have to be modeled. Of course, the time taken for the transistor-level simulations can be reduced by parallelizing the modeling process. But this modeling time can be further reduced by decomposing the considered circuit into blocks which already have been modeled. On the one hand, certain circuits, such as the multiplexer used as case study in Section 7, present a repeating structure. Therefore, the circuit can be decomposed into a minimum number of different blocks. On the other hand, analog circuits usually make used of common circuitry such as current mirrors or differential pairs. These blocks can be modeled and stored into a library of reuseable models. Furthermore, the same can be done for the faults inside these blocks. The fault sensitization step can be carried out on beforehand, so that the test generation procedure can directly proceed to the backtracing step.

\subsection{Test Compression}

In the scope of this work, it was proposed to stop the algorithm as soon as a test signal able to detect a given fault is calculated. Therefore, if multiple faults have to be detected, and test generation steps are carried out independently, as many test signals as the number of faults will be generated. However, in this broader scope consisting in generating a set of signals detecting a set of faults, it can be interesting to let the algorithm calculate for each fault the whole range of test signals detecting the given fault.
By calculating the whole set of solution for every fault, the intersections between these solutions can be searched. If an intersection between two solutions exists, a test signal able to detect these two faults can be found and the total number of needed tests can be reduced.

\section{Industrial case study}

In order to demonstrate the proposed method in practice, the whole workflow presented in this paper has been applied to a circuit taken from an industrial integrated circuit. The schematic diagram of the circuit under consideration is shown in Figure 12. This multiplexer circuit is the first link in the front end of an analog signal processing chain. It presents three analog inputs (in1,in2,in3), two analog outputs which cannot be accessed (out1 and out2) and seven configuration bits controlling the state of the transmission gates $\left(\mathrm{s}_{11}, \ldots, \mathrm{s}_{71}\right)$. Additionally, test circuitry has been added in the design to access the signals during test mode; leading to two extra analog outputs (test1 and test2). In total, the whole circuit presents 50 MOSFET transistors.

After applying the 5-fault model introduced in Section 2 and removing the redundant faults, a list of 204 faults was generated. Transistor-level simulations show that a fault coverage of $45.6 \%$ is achieved by the dedicated specificationbased tests, where the fault coverage is calculated as the ratio between the number of detected faults and the total number of faults. The steps of the automatic workflow were applied to the circuit in order to generate test signals. First, the circuit partitioning decomposed the circuit into 15 blocks interconnected by 18 nodes, and these were modeled by piecewise linear approximations. Then, the computation phase was carried out for the faults undetected by the specification-based tests.

In one case, the undetected fault was an open drain in the nMOS transistor of the transmission gate controlled by $\mathrm{s}_{11}$. The fault sensitization step found that a difference can be activated if the transmission gate is conducting and that two 
low voltages are imposed on both ends of the transmission gate with a voltage difference. In such a configuration, the pMOS transistor is in the cut-off region and the nMOS transistor conducts current in the good case. But an open drain fault prevents the transistor to conduct and therefore the fault is sensitized. The backtracing step generated a signal applying sensitizing conditions by activating the transmission gates controlled by $s_{11}$ and $s_{21}$, and by applying $0.5 \mathrm{~V}$ on in 1 and $1.2 \mathrm{~V}$ on in2.

Finally, it was found that the proposed method can improve the fault coverage of the considered circuit up to $98 \%$. An analysis of the remaining faults confirmed that these ones cannot be caught with DC signals without extra DfT. An example of such a fault is an open source on the nMOS transistor used to ground the node between the two transmission gates of the test circuitry. That fault can only be sensitized when the transistor is activated and a voltage is imposed on its drain. However, the digital control of the transistors does not allow to realize such a configuration since this transistor is supposed to ensure the connection to ground only when the two surrounding transmission gates are closed. Therefore, the fault cannot be detected with DC signals without extra DfT.

\section{Conclusion}

In this paper, a method has been presented for the generation of DC test stimuli targeting a given fault for a given circuit. The developed procedure allows to handle analog circuits without restriction on the nonlinearities they present. By dividing the considered circuit into sub-circuits, the test generation problem has been transformed into the resolution by interval analysis of Kirchhoff's law for a set of interconnected blocks. Hence, the method allows to cope with industrial circuits presenting multiple inputs/outputs. Finally, due to the properties of the interval analysis, the method can state whether a fault can be detected with DC signals without extra DfT circuitry.

The different steps involved in the proposed method have been illustrated on basic circuits to build up an insight into the core ideas. Then, the method has been applied to an industrial mixed-signal circuit in order to demonstrate its efficiency. It has been shown that a fault coverage of $98 \%$ is achieved and that the remaining faults require extra DfT in order to be detected.

\section{References}

[1] J. P. Roth, "Diagnosis of Automata Failures: A Calculus and a Method," IBM Journal of Research and Development, vol. 10, no. 4, pp. 278-291, July 1966.

[2] M. Bushnell and V. D. Agrawal, Essentials of Electronic Testing for Digital, Memory and Mixed-Signal VLSI Circuits. Springer Science \& Business Media, 2000, vol. 17.
[3] W. Dobbelaere, R. Vanhooren, A. Coyette, and G. Gielen, "Analog Fault Coverage Improvement Using Defect-Specific Masking," pp. $1-6,2015$.

[4] M. Soma, "Structure and Concepts for Current-Based Analog Scan," pp. 517-520, May 1995.

[5] B. Burdiek, "Generation of Optimum Test Stimuli for Nonlinear Analog Circuits Using Nonlinear-Programming and Time-Domain Sensitivities," pp. 603-609, 2001.

[6] R. Ramadoss and M. L. Bushnell, "Test Generation for Mixed-Signal Devices Using Signal Flow Graphs," Journal of Electronic Testing, vol. 14 , no. 3, pp. 189-205, 1999.

[7] M. Stančić and H. G. Kerkhoff, "Testability-analysis driven testgeneration of analogue cores," Microelectronics journal, vol. 34, no. 10, pp. 913-917, 2003.

[8] H. G. Kerkhoff, "The test search for true mixed-signal cores," Microelectronics journal, vol. 36, no. 12, pp. 1103-1111, 2005.

[9] F. C. M. Kuijstermans, M. Sachdev, and A. P. Thijssen, "DefectOriented Test Methodology for Complex Mixed-Signal Circuits," pp. $18-23,1995$

[10] A. Coyette, B. Esen, R. Vanhooren, W. Dobbelaere, and G. Gielen, "Automated Testing of Mixed-Signal Integrated Circuits by Topology Modification," pp. 1-6, April 2015.

[11] S. Sunter, "Experiences with an industrial analog fault simulator and engineering intuition," pp. 1-5, June 2015.

[12] T. Olbrich, J. Pérez, I. A. Grout, A. M. Richardson, and C. Ferrer, "Defect-oriented vs schematic-level based fault simulation for mixedsignal ICs," pp. 511-520, 1996.

[13] H. G. Stratigopoulos, M. J. Barragan, S. Mir, H. L. Gall, N. Bhargava, and A. Bal, "Evaluation of Low-Cost Mixed-Signal Test Techniques for Circuits with Long Simulation Times," pp. 1-7, Oct 2015.

[14] K. Deb, Multi-Objective Optimization Using Evolutionary Algorithms. John Wiley \& Sons, 2001, vol. 16.

[15] S. Bhunia, A. Raychowdhury, and K. Roy, "Defect Oriented Testing of Analog Circuits Using Wavelet Analysis of Dynamic Supply Current," Journal of Electronic Testing, vol. 21, no. 2, pp. 147-159, 2005

[16] I. Kossaczkỳ, "A recursive approach to local mesh refinement in two and three dimensions," Journal of Computational and Applied Mathematics, vol. 55, no. 3, pp. 275-288, 1994.

[17] J. Huerta, M. Chover, R. Quirós, R. Vivó, and J. Ribelles, "Binary Space Partitioning Trees: A Multiresolution Approach,” pp. 148-154, 1997.

[18] T. Kanungo, D. M. Mount, N. S. Netanyahu, C. D. Piatko, R. Silverman, and $\mathrm{A}$. $\mathrm{Y}$. Wu, "An efficient k-means clustering algorithm: analysis and implementation," IEEE Transactions on Pattern Analysis and Machine Intelligence, vol. 24, no. 7, pp. 881-892, Jul 2002.

[19] L. Jaulin, Applied Interval Analysis: with Examples in Parameter and State Estimation, Robust Control and Robotics. Springer Science \& Business Media, 2001, vol. 1.

[20] K. Yamamura and K. Suda, "An efficient and practical algorithm for finding all dc solutions of nonlinear circuits," pp. 1111-1115, 2007

[21] D. Sotiropoulos and T. Grapsa, "An Interval Branch-and-Bound Algorithm for Global Optimization using Pruning Steps," vol. 2, pp. 455-459, 2001. 